\title{
Experimental Results of the EU ITER Prototype Gyrotrons
}

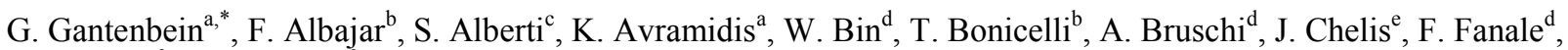 \\ F. Legrand ${ }^{\mathrm{f}}$, V. Hermann ${ }^{\mathrm{f}}$, J.-P. Hogge ${ }^{\mathrm{c}}$, S. Illy ${ }^{\mathrm{a}}$, Z. C. Ioannidis ${ }^{\mathrm{a}}$, J. Jin', J. Jelonnek ${ }^{\mathrm{a}}$, W. Kasparek ${ }^{\mathrm{g}}$, G. P. Latsas ${ }^{\mathrm{e}}$, \\ C. Lechte ${ }^{\mathrm{g}}$, M. Lontano ${ }^{\mathrm{c}}$, I. G. Pagonakis ${ }^{\mathrm{a}}$, T. Rzesnicki ${ }^{\mathrm{a}}$, C. Schlatter ${ }^{\mathrm{c}}$, M. Schmid ${ }^{\mathrm{a}}$, I. G. Tigelis ${ }^{\mathrm{e}}$, M. Thumm $^{\mathrm{a}}$, \\ M. Q. Tran ${ }^{\mathrm{c}}$, J. L. Vomvoridis ${ }^{\mathrm{h}}$, A. Zein ${ }^{\mathrm{a}}$, A. Zisis ${ }^{\mathrm{e}}$
}

${ }^{a}$ Karlsruhe Institute of Technology (KIT), IHM, Kaiserstr. 12, 76131 Karlsruhe, Germany

${ }^{\mathrm{b}}$ European Joint Undertaking for ITER and the Development of Fusion Energy (F4E), Barcelona, E-08019, Spain

${ }^{\mathrm{c}}$ Swiss Plasma Center (SPC), Ecole Polytechnique Fédérale de Lausanne, CH-1015 Lausanne, Switzerland

${ }^{\mathrm{d}}$ Istituto di Fisica del Plasma, Consiglio Nazionale delle Ricerche, Via R.Cozzi 53, 20125 Milano, Italy

${ }^{\mathrm{e}}$ Faculty of Physics, National and Kapodistrian University of Athens, Zografou, GR-157 84, Athens, Greece

${ }^{f}$ Thales Electron Devices, 2 rue Marcel Dassault, Vélizy-Villacoublay, F-78141, France

${ }^{\mathrm{g}}$ University of Stuttgart, IGVP, Pfaffenwaldring 31, 70569 Stuttgart, Germany

${ }^{\mathrm{h}}$ National Technical University of Athens, Greece

\begin{abstract}
The European $1 \mathrm{MW}, 170 \mathrm{GHz} \mathrm{CW}$ industrial prototype gyrotron for ECRH\&CD on ITER was under test at the KIT test facility during 2016. In order to optimize the gyrotron operation, the tube was thoroughly tested in the short-pulse regime, with pulse lengths below $10 \mathrm{~ms}$, for a wide range of operational parameters. The operation was extended to longer pulses with a duration of up to $180 \mathrm{~s}$. In this work we present in detail the achievements and the challenges that were faced during the long-pulse experimental campaign.
\end{abstract}

\section{Introduction}

The European $1 \mathrm{MW}, 170 \mathrm{GHz}$ industrial prototype CW gyrotron for ECRH\&CD on ITER is a conventional (hollow-cavity) gyrotron, which has been developed by the European GYrotron Consortium (EGYC) in cooperation with the industrial partner Thales Electron Devices (TED), under the coordination of the European Joint Undertaking for ITER and the Development of Fusion Energy (F4E).

According to the EU development strategy, the design of the CW gyrotron is completely based on a corresponding modular short-pulse (SP) prototype and the $1 \mathrm{MW}, 140 \mathrm{GHz}$ gyrotron development for W7-X [1]. The SP prototype was developed in order to validate the scientific design in terms of RF output power, total efficiency and quality of RF output beam.

The SP prototype was extensively tested at the KIT test facility in 2015 and the scientific design of the individual tube components has been verified, with the gyrotron delivering an output power of higher than $1.2 \mathrm{MW}$ with $40 \%$ total efficiency (in single-stage depressed collector operation) and with a RF output beam Gaussian mode content of about $~ 98 \%$ [2].

The CW industrial prototype was delivered to the KIT test facility in early 2016 and the experiments started immediately in the short-pulse regime (with pulse length below $10 \mathrm{~ms}$ ). The main goal of this first validation phase was to optimize the gyrotron alignment with respect to the magnetic field axis, as well as to determine the best operational parameters, namely accelerating voltage, beam current and magnetic field profile, in terms of generated RF power. A summary of the first short-pulse experiments at KIT with the CW prototype is presented in $[3,4]$.

The optimal operational parameters at short-pulses were used as a starting set for the conditioning of the $\mathrm{CW}$ tube with $\mathrm{RF}$ and the progressive increase of the pulse length. After some weeks of conditioning, it was possible to operate the gyrotron with pulse lengths of up to $180 \mathrm{~s}$, which is the limit at the High-Voltage (HV) power supply currently available at KIT.

In this work we focus on the performance of the tube during the second validation phase, i.e. long-pulse operation at the KIT test facility, details regarding the operating points are presented, the performance of the tube during the short-pulse phase of the experiments is summarized and the long-pulse phase of the experiments with pulses up to $180 \mathrm{~s}$ is analyzed.

Corresponding author: gerd.gantenbein@kit.edu 


\section{Operating Points for the CW Prototype}

In order to have higher flexibility in the operation of the $\mathrm{CW}$ gyrotron, two operating points in terms of the accelerating voltage have been defined. The nominal one is the High Voltage Operating Point (HVOP), which corresponds to an accelerating voltage of $79.5 \mathrm{kV}$ and a beam current of $40 \mathrm{~A}$, the alternative one is the Low Voltage Operating Point (LVOP), which corresponds to an accelerating voltage of $71 \mathrm{kV}$ and a beam current of $45 \mathrm{~A}$. Further details regarding the operational parameters of both operating points, as well as the corresponding simulated performance of the gyrotron, are given in Table 1.

The optimization of the gyrotron operation in the short-pulse regime started and focused on the LVOP, whereas only brief experiments with the HVOP were performed. Due to the limited time of the experimental campaign the experiments in the long-pulse regime were continued in the LVOP, as the tube was specifically conditioned for such operating parameters. In the following paragraphs the presented results refer always to the LVOP, except if it is stated differently.

Table 1. Operating points for $\mathrm{CW}$ operation

\begin{tabular}{|c|c|c|}
\hline Parameter & HVOP & LVOP \\
\hline Operating mode & $\mathrm{TE}_{32,9}$ & $\mathrm{TE}_{32,9}$ \\
\hline Cavity magnetic field & $6.78 \mathrm{~T}$ & $6.69 \mathrm{~T}$ \\
\hline Accelerating voltage & $79.5 \mathrm{kV}$ & $71.0 \mathrm{kV}$ \\
\hline Depression voltage & $35 \mathrm{kV}$ & $30 \mathrm{kV}$ \\
\hline Beam current $\mathrm{I}_{\mathrm{b}}$ & $40 \mathrm{~A}$ & $45.3 \mathrm{~A}$ \\
\hline Cavity beam radius $\mathrm{R}_{\mathrm{b}}$ & $9.44 \mathrm{~mm}$ & $9.44 \mathrm{~mm}$ \\
\hline Cavity pitch factor $\alpha$ & 1.29 & 1.22 \\
\hline Output power at window & $1 \mathrm{MW}$ & $1 \mathrm{MW}$ \\
\hline Frequency & $170.23 \mathrm{GHz}$ & $170.23 \mathrm{GHz}$ \\
\hline Interaction efficiency & $35 \%$ & $35 \%$ \\
\hline $\begin{array}{c}\text { Total efficiency } \\
\text { w/o depressed collector }\end{array}$ & $32 \%$ & $32 \%$ \\
\hline $\begin{array}{c}\text { Total efficiency } \\
\text { w/ depressed collector }\end{array}$ & $>50 \%$ & $>50 \%$ \\
\hline Peak cavity wall loading & $2.1 \mathrm{~kW} / \mathrm{cm}^{2}$ & $2.1 \mathrm{~kW} / \mathrm{cm}^{2}$ \\
\hline
\end{tabular}

\section{Short-Pulse Operation}

A basic description of the performance of the tube in SP operation is presented in [3-4]. Herein, we report on further details for the operation of the $\mathrm{CW}$ gyrotron in the SP regime. It should be noted that the gyrotron performance described in this section corresponds to pulses not longer than $5 \mathrm{~ms}$ and always to non-depressed collector operation of the tube.

The alignment verification of the gyrotron cavity with respect to the magnetic field has been performed in the short-pulse regime using a method developed at KIT, which is based on the study of symmetry in the excitation regions of the main mode [5]. The concept is to introduce a radial displacement of the electron beam in the cavity area using a set of super-conducting (SC) transverse field dipole coils integrated inside the KIT magnet and monitor the influence of this displacement on the excitation of a cavity mode. By changing the current of the dipole coils, the switching point between the main mode and its neighboring competitors is recorded. The graphical representation of the switching points forms a circle, around the dipole currents values, where the main mode is properly excited. The distance from the center of this circle to the origin of the dipole coils coordinate system (zero current in both directions) provides an indication regarding the misalignment of the magnetic field axis with respect to the gyrotron cavity axis.

During the alignment phase it was found that these current values correspond roughly to a $(0.56 \mathrm{~mm}$, $0.41 \mathrm{~mm}$ ) shift of the electron beam. By operating the gyrotron at the LVOP with the above mentioned coil current values, the maximum RF output power obtained with near nominal voltage/current parameters was $930 \mathrm{~kW}$ with $26 \%$ total efficiency (in non-depressed collector operation). More details about the gyrotron performance under these conditions are given in [4].

The thermal footprint of the gyrotron output RF beam has been imaged on two different target plates of $200 \mathrm{~mm}$ diameter, one made from PVC and a second one from regular paper. The plates are placed along the RF beam path at various distances between $180 \mathrm{~mm}$ and $1300 \mathrm{~mm}$ from the gyrotron output window. The distance was progressively increased using a motorized movement stage. According to the post processing of the measurements with a phase retrieval algorithm, the Gaussian mode content of the beam was found to be at least $97 \%$, which fulfills the ITER specifications.

\section{Long-Pulse Operation}

In order to proceed from the short-pulse operation to $180 \mathrm{~s}$ long-pulse operation, several weeks were needed including the optimization of the test-stand auxiliaries as well as of the control system. Recall that due to limitations of the HV power supply at KIT, the maximum pulse length that can be achieved with beam current up to $50 \mathrm{~A}$ is $180 \mathrm{~s}$ with a $1 / 10$ duty cycle. Due to these time limitations the experimental campaign was focused on the LVOP (beam current $\sim 45 \mathrm{~A}$ and accelerating voltage $\sim 71 \mathrm{kV}$ ).

Fig. 1 presents the control system recordings for one of the first $180 \mathrm{~s}$ pulses that were achieved. In this figure the black curve corresponds to the accelerating voltage, which is the sum of the cathode voltage (green curve) and the depression voltage (red curve), whereas the blue curve is the emitted electron beam current. In the current curve it is obvious that during the first seconds of the pulse the emitted current is slightly reduced because of the cathode cooling effect and then recovers towards the targeted value. This recovery is achieved by employing a current boosting scheme, which controls the filament current before and during the pulse. 
In the same figure, the olive and yellow curves correspond to the vacuum pressure signal of the ion getter pumps. During most of the pulses including this specific one, the vacuum level remained better than $10^{-}$ ${ }^{8}$ mbar. The purple curve represents the output voltage of the mode-loss RF diode, which deactivates the gyrotron operation as soon as the signal drops below a predefined level. This could happen for example in case that the accelerating voltage was increased above the value which the nominal operating mode could tolerate. In general, no unexpected mode excitation took place during the experimental campaign.

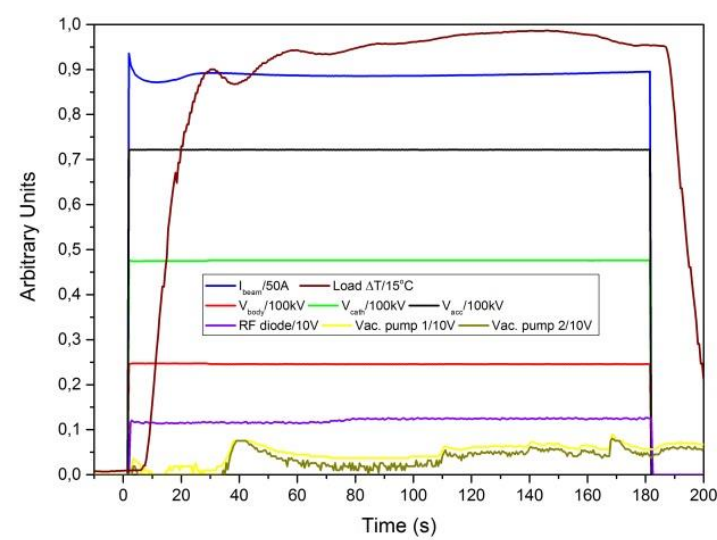

Fig. 1. Typical $180 \mathrm{~s}$ pulse achieved during the experiments, the temperature measurement in the load (proportional to RF power) is delayed and shows oscillations at the beginning of the pulse due to the KIT cooling system only (all measurements normalised to the indicated values).

The generated power and the dissipated losses on the internal components of the gyrotron as well as of the auxiliaries in the microwave box are monitored through the various cooling circuits that are connected to a calorimetry system.

Table 2 presents an example of the power that was measured in the different circuits of the calorimetry system during a typical $0.8 \mathrm{MW}$ pulse. It should be noted that the $\mathrm{CW}$ gyrotron prototype is equipped with three cooling circuits for the beam tunnel, the cavity and the quasi-optical launcher. These circuits also support the cooling of internal mirror 1 (M1) and mirror 2 (M2) of the quasi-optical system of the gyrotron, which are connected in series. The last mirror of the system, namely mirror 3 (M3) has a completely individual cooling circuit.

Considering that the losses on M1 and M2 should be comparable to those on M3, it is evident from Table 2 that the measured losses in the beam tunnel were negligible (the accuracy of the calorimetric measurements is in the order of $1 \%$ ).

In the last column of the table the power measurements are expressed with respect to the total input power of the electron beam, that is calculated by the cathode voltage and the beam current, which for this pulse were $47.4 \mathrm{kV}$ (depressed collector operation of the gyrotron) and $47.5 \mathrm{~A}$, respectively. The internal losses (internal components of the tube) are in the level of $5 \%$ with respect to the RF power at the synthetic diamond window, whereas the external losses (inside the microwave box) are in the range of 2-3\% with respect to the power at the window.

Table 2. Calorimetry measurements for a typical pulse

\begin{tabular}{|l|l|l|}
\hline Measurement & $\begin{array}{l}\text { Power } \\
\text { (kW) }\end{array}$ & \% of input power \\
\hline Cavity (M1-M2) & 28 & 1.24 \\
\hline Beam tunnel (M1-M2) & 1.26 & 0.06 \\
\hline Launcher (M1-M2) & 11 & 0.49 \\
\hline Mirror 3 (M3) & 1 & 0.04 \\
\hline Window & 1 & 0.04 \\
\hline Mirror box & 3 & 0.13 \\
\hline Relief load & 2 & 0.09 \\
\hline Collector & 1365 & 60.67 \\
\hline Water load & 792 & 35.20 \\
\hline Microwave box & 21 & 0.93 \\
\hline Total & 2225 & 99 \\
\hline
\end{tabular}

Setting properly the three independent currents of the gyrotron magnet (Oxford Instruments), a quite wide area of magnetic field angles $\varphi_{\mathrm{B}}$ at the cathode emitter and beam radii $R_{b}$ in the cavity was examined. The magnetic field angle $\varphi_{\mathrm{B}}$ is an important operating parameter that is strongly related to the pitch factor of the electrons [6].
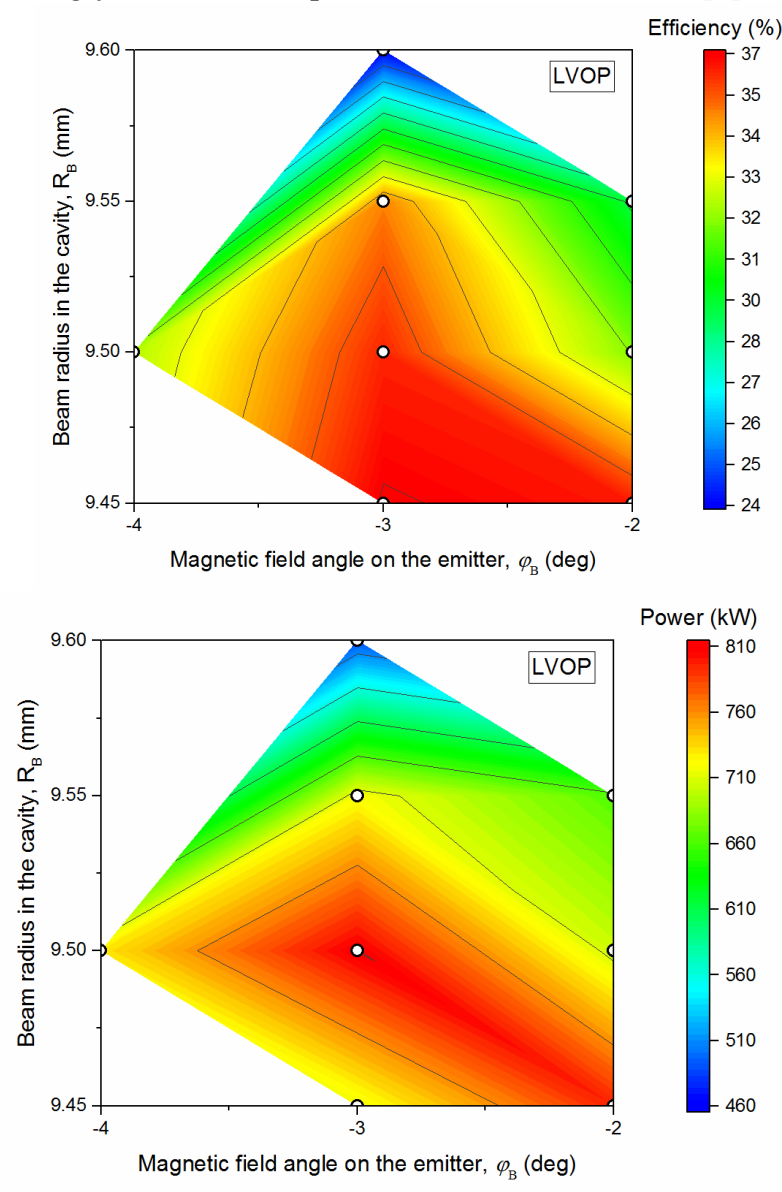

Fig. 2. Operational map - RF power (bottom) and total efficiency (top) with respect to the magnetic field angle at the cathode emitter and the radius of the electron beam in the cavity. 
Fig. 2 presents the operational maps of the gyrotron in long-pulse operation in dependence of $\varphi_{B}$ and $R_{b}$. In these figures the white circles correspond to the position where the actual measurements took place. It should be noted that the performance reported for each $\left(\varphi_{B}, R_{b}\right)$ combination corresponds to the optimal voltage and beam current operating parameters found at this point, whereas the collector depression voltage is usually set in the range $20-25 \mathrm{kV}$ without special optimization.

The optimal performance of the gyrotron in terms of output power was found at the operating point $\left(\varphi_{B}=-3^{\circ}\right.$, $\mathrm{R}_{\mathrm{b}}=9.50 \mathrm{~mm}$ ), where $800 \mathrm{~kW}$ were generated in most of the pulses. The maximum RF power value recorded is $811 \mathrm{~kW}$ with a total efficiency of $36 \%$ (in depressed collector operation).

Fig. 3 shows the RF power and the corresponding total efficiency versus the collector depression voltage for the operating point $\left(\varphi_{B}=-3^{\circ}, R_{b}=9.50 \mathrm{~mm}\right)$. The measurements were recorded at $60 \mathrm{~s}$ long pulses. By increasing the depression voltage, the generated RF power remains almost constant, while the efficiency practically increases linearly, reaching a maximum value of $38 \%$.

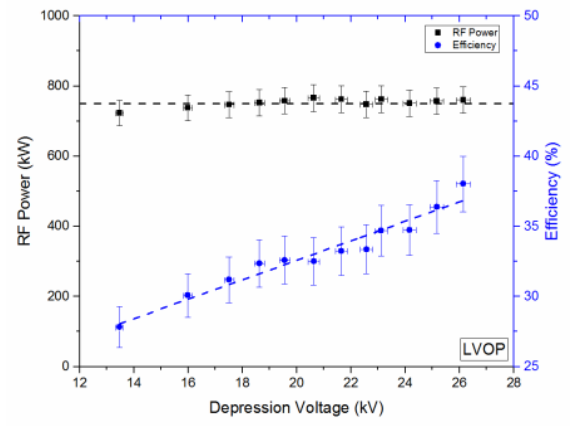

Fig. 3. RF power and corresponding total efficiency versus the depression voltage for the LVOP at the operating point $\left(\varphi_{B}=-3^{\circ}, R_{b}=9.50 \mathrm{~mm}\right)$.

The maximum value of the applied depression voltage, where stable operation of the gyrotron was achieved is $26 \mathrm{kV}$. The operation with higher values of the depression voltage was quite challenging, since a body current in the $\mathrm{mA}$ range and often instabilities during the first seconds of the pulses were observed. Such instabilities, which include arcing and beam current fluctuations, could be an indication of an increased number of reflected electrons [7].

The time dependency of the frequency of the nominal mode $\mathrm{TE}_{32,9}$ was recorded using the KIT pulse shape analysis (PSA) measurement system [8].

Starting from approximately $170.300 \mathrm{GHz}$ the operating frequency drops to $169.950 \mathrm{GHz}$ $(\Delta \mathrm{f}=350 \mathrm{MHz})$ within $500 \mathrm{~ms}$ at an output power of $650-700 \mathrm{~kW}$. The frequency and frequency drop meet very well the ITER requirement of $170 \mathrm{GHz} \pm 0.3 \mathrm{GHz}$.

The above described frequency drop during a pulse is, in general, attributed to the neutralization of the electron beam as well as to the thermal expansion of the cavity that strongly depends on the generated RF power.
A very brief part of the experimental campaign was focused on long-pulse operation of the tube at the HVOP with pulses of $30 \mathrm{~s}$ and $60 \mathrm{~s}$ duration. A RF power above $800 \mathrm{~kW}$ was generated with the corresponding total efficiency of approximately $35 \%$ (in depressed collector operation).

\section{Conclusion}

The first phase of the experiments with the $1 \mathrm{MW}$, $170 \mathrm{GHz} \mathrm{CW}$ prototype EU gyrotron for the ITER ECRH\&CD system have been successfully completed at KIT. With these experiments most of the ITER specifications have been verified including the $\mathrm{HV}$ properties of the gyrotron, the frequency of the nominal mode, the stability of the vacuum, as well as the $\mathrm{TEM}_{00}$ mode purity (Gaussian content higher than $97 \%$ ) and the alignment of the RF beam at the diamond window. The maximum RF power that was achieved in $180 \mathrm{~s}$ pulses is $0.8 \mathrm{MW}$ both for the LVOP and HVOP with total efficiency $38 \%$ and $35 \%$, respectively. Considering that this is the first industrial $\mathrm{CW}$ prototype of the tube, the achieved performance in the first phase of the experiments is very good.

The experiments will be continued at SPC, Lausanne. The gyrotron was transported to SPC in March 2017 and installed in the specified (Cryogenic) magnet. Taking advantage of the flexibility of the High Voltage Power Supply (HVPS) at SPC, the goal is to further increase the pulse length. Furthermore, reliability and power modulation tests will be performed. The goal is to achieve at least $95 \%$ success rate out of 50 subsequent $180 \mathrm{~s}$ pulses. Regarding the modulation tests (up to 5 $\mathrm{kHz}$ ), $100 \%$ power depth modulation using both the main and the body HVPS will be attempted during $180 \mathrm{~s}$ pulses.

\section{Acknowledgement}

This work is partly supported by Fusion for Energy under Grant F4E-GRT-553 to the European Gyrotron Consortium (EGYC). EGYC is a collaboration among SPC, Switzerland; KIT, Germany; HELLAS, Greece; IFP-CNR, Italy. The views expressed in this publication are the sole responsibility of the authors and do not necessarily reflect the views of F4E and the European Commission.

\section{References}

[1] G. Gantenbein, et al., J. Infrared Milli Terahertz Waves, 32, no. 3, pp. 320-328, Dec. 2011.

[2] T. Rzesnicki, et al., IRMMW-THz 2015, proc. 3134554, Hong Kong, Aug. 23-28, 2015.

[3] J.-P. Hogge, et al., IRMMW-THz 2016, Copenhagen, Denmark, Sep 25-30, 2016.

[4] T. Rzesnicki, et al., Fusion Eng. Des., published on-line, Feb. 2017. [5] T. Rzesnicki, et al., 37th International Conference on Infrared, Millimeter, and Terahertz Waves, NSW, Australia, 2012.

[6] I. Gr. Pagonakis, et al., Phys. Plasmas, 24, no. 3, 2017.

[7] I. Gr. Pagonakis, et al., 9th Int. Workshop "Strong Microwaves and Terahertz Waves: Sources and Applications", Nizhny Novgorod, Russia, 24-30 July 2014.

[8] A. Schlaich, et al., IEEE Trans. Microwave Theory Tech., 61, no. 12, pp. 4660-4666, Dec 2013. 